\title{
The Oxford Modular Cataract Image Analysis System
}

\author{
JOHN M. SPARROW, NICHOLAS A. P. BROWN, G. ADRIEN SHUN-SHIN, \\ ANTHONY J. BRON \\ Oxford
}

\begin{abstract}
Summary
A modular system of acquisition and analysis of Scheimpflug, retro-illumination and fluorescence images of the in vivo human crystalline lens is described. Image analysis is directed towards the following goals: Scheimpflug slit-images are analysed for: (1) The optical density of nuclear cataract present; (2) The dimensions of the lens and the lenticular zones; (3) The curvatures of the lens and lenticular zones.

Retro-illumination images are analysed for: (1) The percentage area which is occupied by cataract; (2) A combined measure (weighted integral) describing both the amount of cataract present and its optical density. Lenticular auto-fluorescence images are analysed for the mean density (fluorescence) of the lens as a whole. A pilot study of the repeatability of the methods is presented.
\end{abstract}

The need for an objective, standardised method of cataract quantification is well recognised. . $2,3,4,5,6,7,8$ Image analysis of Scheimpflug lens photographs is a well established technique in clinical lens research..$^{9.10}$ More recently improved quality retro-illumination lens images have become available, ${ }^{11,12,13}$ which have encouraged serious attempts at objective analysis of these in vivo images. ${ }^{14,15,16,17,18,19}$ Lens photographs have in addition been subjected to fluorescence anal$y \operatorname{sis}^{20,21}$ and to colour analysis..$^{22.23}$ The analysis system described here was developed by the Clinical Cataract Research Unit, University of Oxford, in conjunction with Pipistrel Research Ltd.*

The system consists of a number of modules which serve image acquisition and analysis (Fig. 1). Photographic systems include Sche- impflug lens photography, ${ }^{9,2+25}$ retro-illumination lens photography, ${ }^{12,13}$ and lenticular auto-fluorescence photography. Charge couple device (CCD) based camera systems have been developed by our laboratory in conjunction with Marcher Enterprises Ltd. $\dagger$ and Pipistrel Research Ltd.* These provide immediate Scheimpflug and retro-illumination images in digital form. Photographic images are transferred to digital form using a CCD camera mounted on a modified photographic slide copier (Bowens Illumitran). Digitised images are analysed using a desktop micro-computer which is fitted with a frame store. The image analysis software has been custom written, and is mouse driven and 'user friendly'. Operating the system requires no expert technical or clinical skills. Large numbers of digitised images can be stored on

From the Clinical Cataract Research Unit, Nuffield Laboratory of Ophthalmology, University of Oxford, Walton Street, Oxford OX2 6AW, England.

*Pipistrel Ltd., Unit 1 Riverview, Walnut Tree Close, Guildford, Surrey, GU1 4UX, England.

$\lceil$ Marcher Enterprises Ltd., 6 Twyford Road, Rotherwas Industrial Est., Hereford, HR2 6JR, England.

Correspondence to: Mr. John M. Sparrow DPhil FRCS FCOphth, Department of Ophthalmology, University of Leicester, Leicester Royal Infirmary, Leicester LE2 7LX, England. 


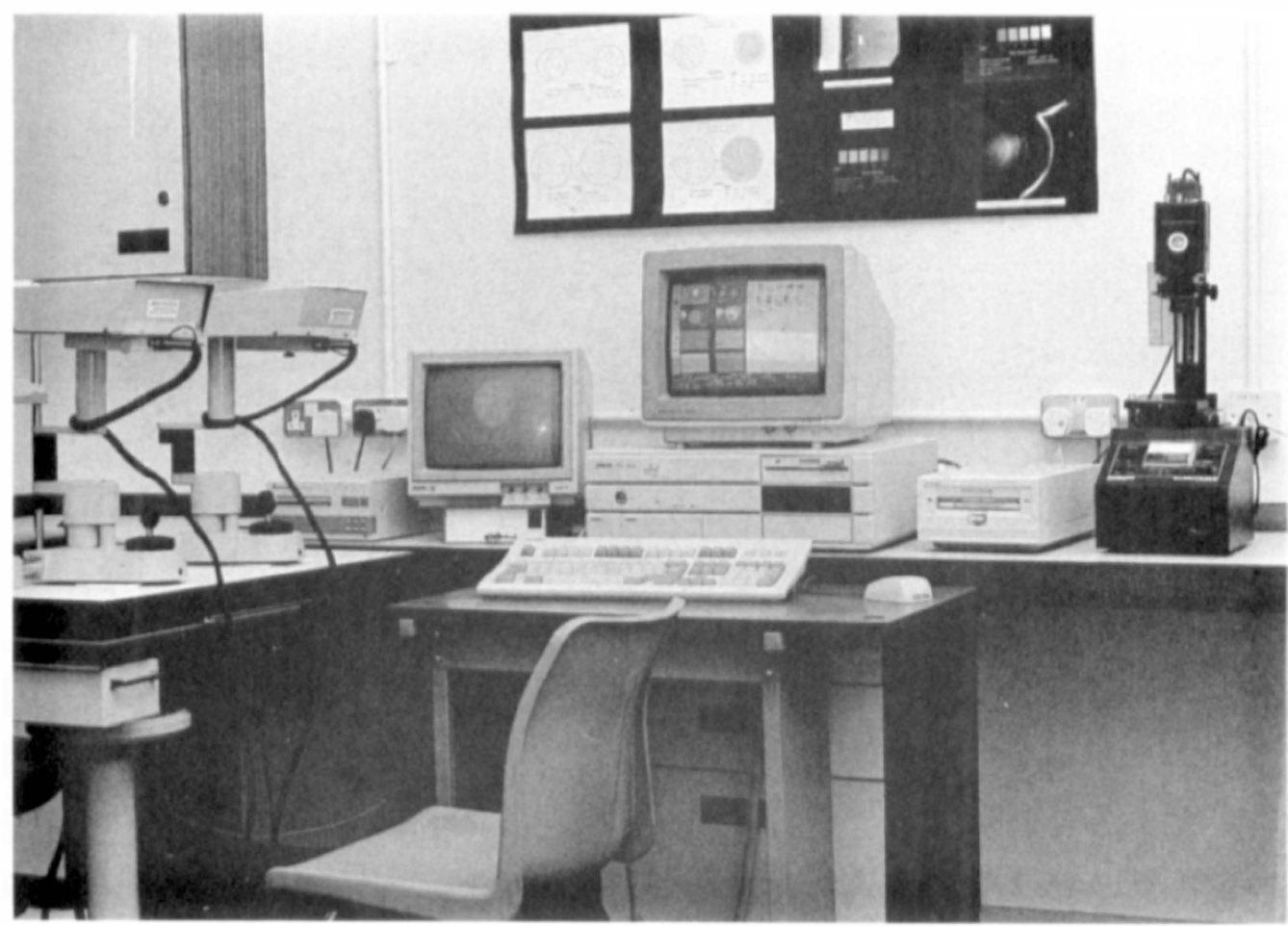

Fig. 1. The components of the Oxford Modular Cataract Image Analysis System. From left to right across the work top: Two CCD camera units for input of Scheimpflug and Retro-illumination images directly from patients, the video printer, the video screen for image display (256 grey levels), the desk top micro-computer with colour monitor, keyboard and mouse, the optical disk drive for image storage, and the 'Bowens' Illumitran with mounted $C C D$ camera for input of photographic images.

optical storage media (over 700 images per optical disk).

\section{Image Acquisition}

Image acquisition may be by either photographic or CCD based camera systems. The Scheimpflug, retro-illumination and autofluorescence photographic systems used in our unit have previously been described, $, 12,13,24,25,26$ and the new generation combined Scheimpflug and retro-illumination CCD cameras will be described in a separate report.

\section{Digital Image Analysis}

Images are loaded into the computer via a standard RS-232 input port. The image analysing computer is an EPSON PC AX with an expanded random access memory (RAM, $2 \mathrm{mb}$ ) in the form of a frame store. The expanded memory is used to store images in digital form, the digital image format being suitable for image analysing procedures. Four images can be held simultaneously in RAM for analysis (further memory expansion will allow an additional 4 images in RAM). The central processing unit of the micro-computer is an 80286 processor, which runs at $10 \mathrm{mHz}$. The machine is sufficiently fast to manipulate images within seconds. There are two screens attached to the computer, one is a standard colour computer screen for control of the software and display of analyses, and the other is a black and white video screen for presentation of the image. The image is displayed on a matrix of $512 \times 512$ picture elements (pixels), with a grey scale range of 256 . The software is mouse driven via icons on the computer screen (Fig. 2), and is composed of a number of sections which are in effect 'tools' for performing various types of digital processing, the operator determining the type of 


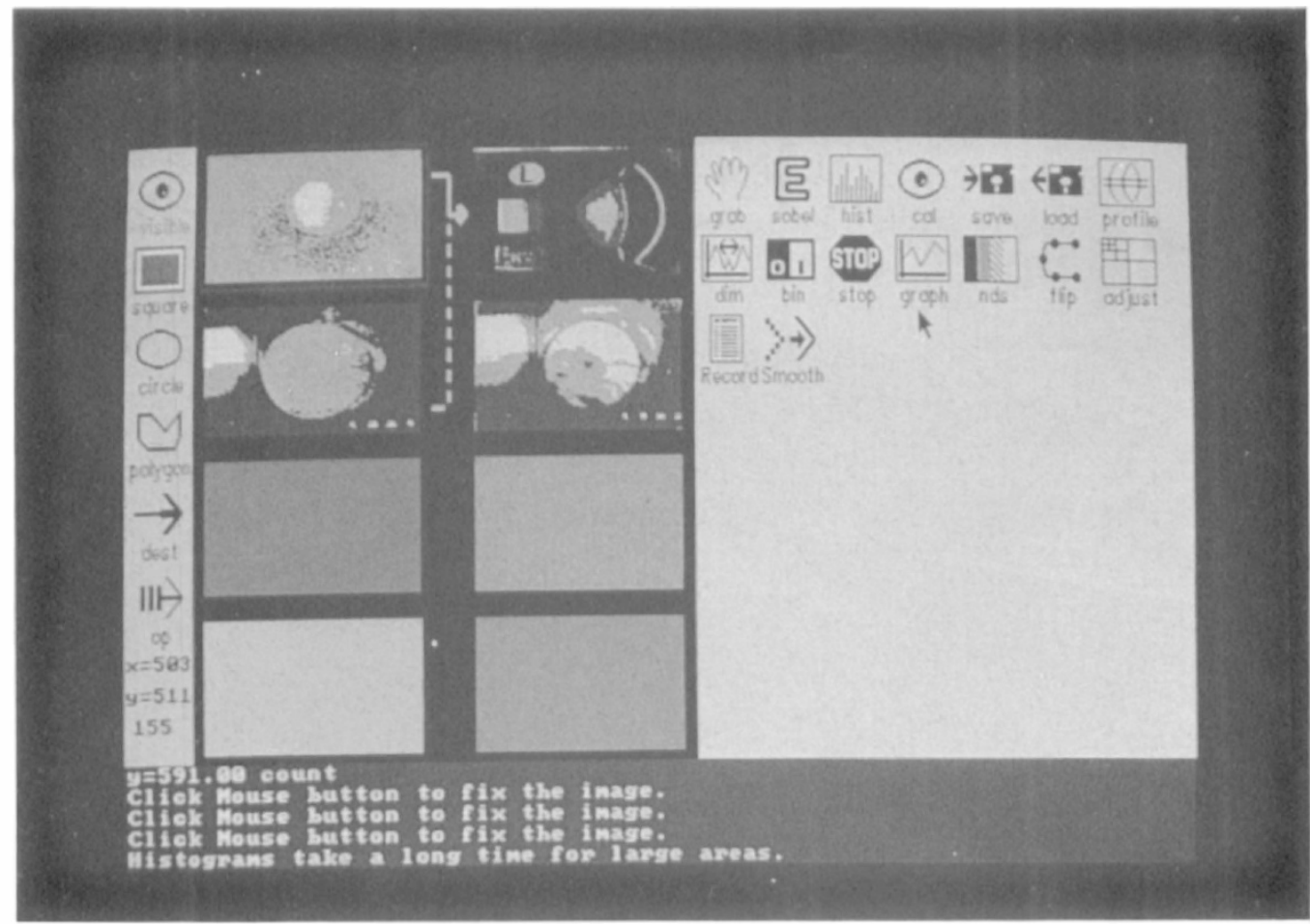

Fig. 2. Computer screen showing presentation of available options for running a digital analysis: Image analysis 'Tools' on the right half, image selection on the left half, and a column for selecting the shape of the 'area of interest' at the extreme left of the screen.

analysis according to the nature of the image under study. Specific image processing procedures have been developed for analysis of Scheimpflug images, retro-illumination images and lenticular auto-fluorescence images. General purpose image processing procedures are also available, such as edge detection, image smoothing and image reversal.

A number of specific, dedicated image processing routines have been developed by the authors, although considerable flexibility exists within the software, which makes it possible to process images according to individual user requirements. (Photographic images obtained from the Topcon SL-45 series of slit-image cameras or Neitz retroillumination camera systems can likewise be processed by the image analysis system). The specific dedicated analyses currently employed in our unit are as follows:

(1) Digital Analysis of Scheimpflug Images

Our analysis of Scheimpflug slit-images is directed towards three goals: Firstly, an assessment of nuclear scatter, secondly measurement of lens dimensions, and thirdly measurement of lens curvatures. A linear density trace running down the axis of the lens is utilised in the first two of these assessments (Fig. 3 and 4). The value of the axial density trace at each point consists of the mean of 10 aligned pixels, thus decreasing the high spatial frequency noise along the trace.

In the assessment of nuclear scatter, the operator makes the measurement by placing the cursor lines at the central nuclear dip in the centre of the lens (Fig. 3 and 4). When no central dip is discernible, the centre of the nucleus is estimated, and this point is chosen for the measurement. (Alternative nuclear locations for density measurement may be selected, our experience however indicates that the centre of the nucleus is least subject to artifactual variability in density). The measurement is made via a calibration routine so that grey levels are calibrated linearly 


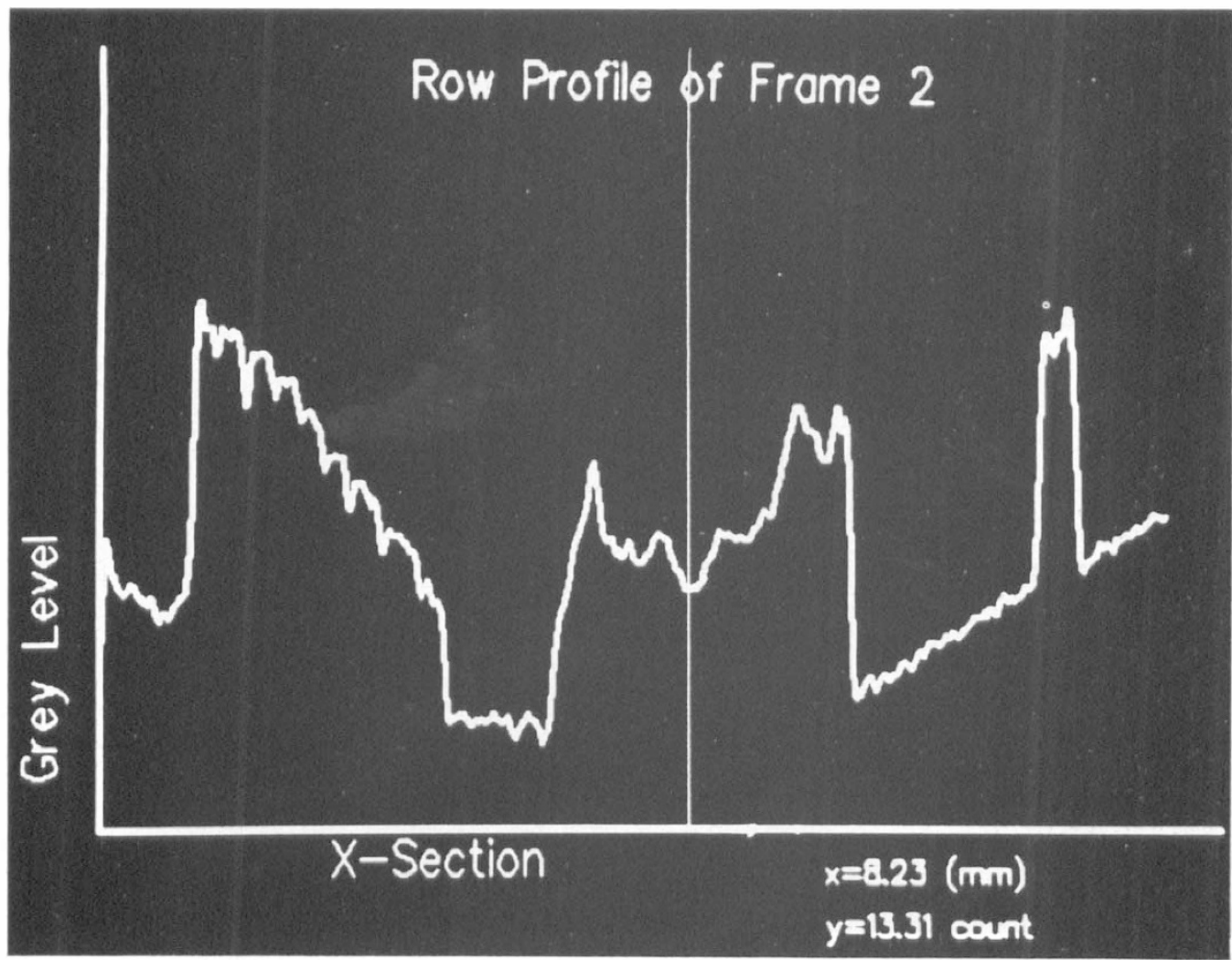

Fig. 3. Screen display of axial linear density trace of Scheimpflug image. The trace passes through the cornea, the lens and the neutral density staircase. The vertical cursor line is positioned at the central nuclear dip (as illustrated also in Fig. 4 for the same image). In this mode calibrated density mesurements are available at any point along the axial trace of the image. Horizontal distance between any two points along the trace are also available, these being calibrated to the original object size.

according to steps on the neutral density 'staircase' (internal standard) on each Scheimpflug image (Fig. 3 and 4). This method takes account of the variability of the density levels in the entire viewing system, and where a photographic camera system is being used, linearity of the response of the photographic emulsion over the range of grey levels being measured is assumed. The assumption of linearity is in general approximately true, because the Scheimpflug camera was designed to operate within the linear portion of the classical sigmoid curve of exposure versus density for photographic emulsion (film type Ilford XP1). ${ }^{9,27}$ Where scatter in other areas of the lens is of interest, these regions can be measured by moving the cursor to the region of interest, the machine displaying automatically the relevant calibrated density value. The units of measurement are standard neutral density units.

Axial lens dimensions are also measured using the linear density trace of the Scheimpflug images. Points for measurement are identified on the trace using the cursor which is displayed simultaneously on both screens, thus allowing judgements on the density trace to be confirmed on the actual image itself (Fig. 3 and 4). The antero-posterior lens thickness, nuclear thickness, anterior clear zone (ACZ) thickness, and anterior chamber depth are measured in this way. Any axial biometric parameter can be measured from the linear density trace, users may thus choose other measures of interest.

Lens curvatures and oblique measures are obtained directly from the image on the video screen by placing a cross shaped $(+)$ cursor at 


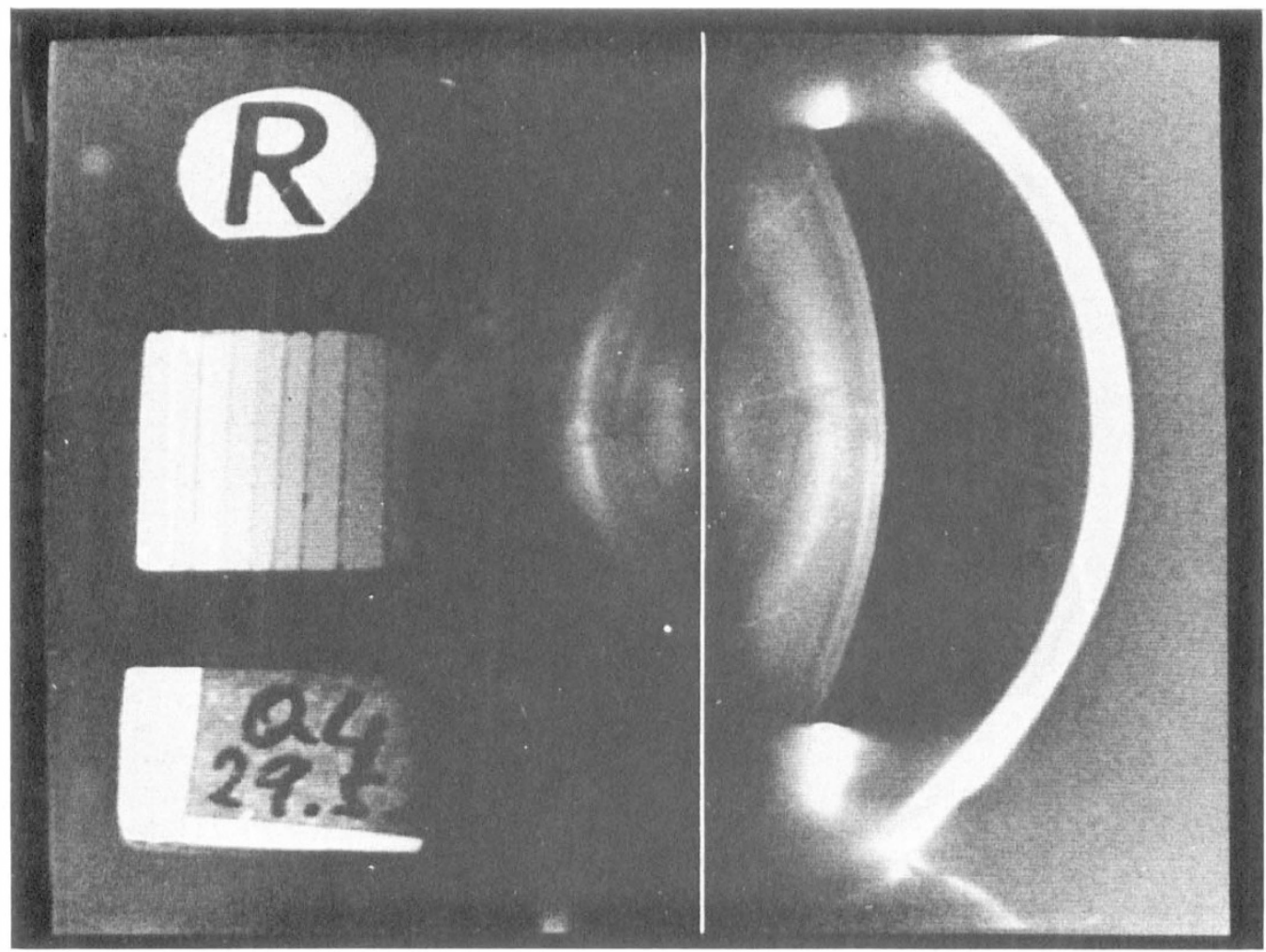

Fig. 4. Scheimpflug image as seen on the video screen, showing cornea, lens and neutral density staircase. In this example the vertical cursor line is positioned at the central nuclear dip (as for the same image on the linear density trace in Fig. 3) By changing to a '+' cursor in this mode, horizontal, vertical and oblique distance measurements are available, as well as measurements of the radius of curvature of any arc in the image. All measurements are calibrated to the original object size.

three points along the curve to be measured. The computer determines the radius of curvature of the arc according to separate vertical and horizontal calibration settings. In this mode vertical or oblique biometric measurements can also be made. Thus the pupil size or other measures of interest are available.

Separate horizontal and vertical calibration is utilised. These calibration values assume spatial uniformity, no corrections being made for horizontal spatial distortion due to either the 'Scheimpflug optics', or the magnification produced by the positive optical power of the cornea and lens of the eye. The spatial distortions from these two sources tend to cancel each other out, the corneal and anterior lenticular effects resulting in increased magnification with increasing distance behind the cornea, while the 'Scheimpflug optics' produce increasing minification with posterior passage. Using a Scheimpflug camera with a horizontal primary magnification of 1.75 to 1 , the net horizontal spatial distortion across an average $(4 \mathrm{~mm})$ lens is aproxiamtely $20 \%$. Scheimpflug cameras with less primary magnification (e.g. 1 to 1 ) would suffer more net horizontal distortion as the optical magnification effect of the eye (approximately 37\% across a $4 \mathrm{~mm}$ lens) is uncompensated by Scheimpflug optics minification. The vertical spatial distortion is small and can be ignored [Sparrow JM Unpublished data].

\section{2) Digital Analysis of Retro-illumination Images}

Analysis of retro-illumination images is aimed at providing an answer to the following questions:

(1) What percentage of the lens in antero- 


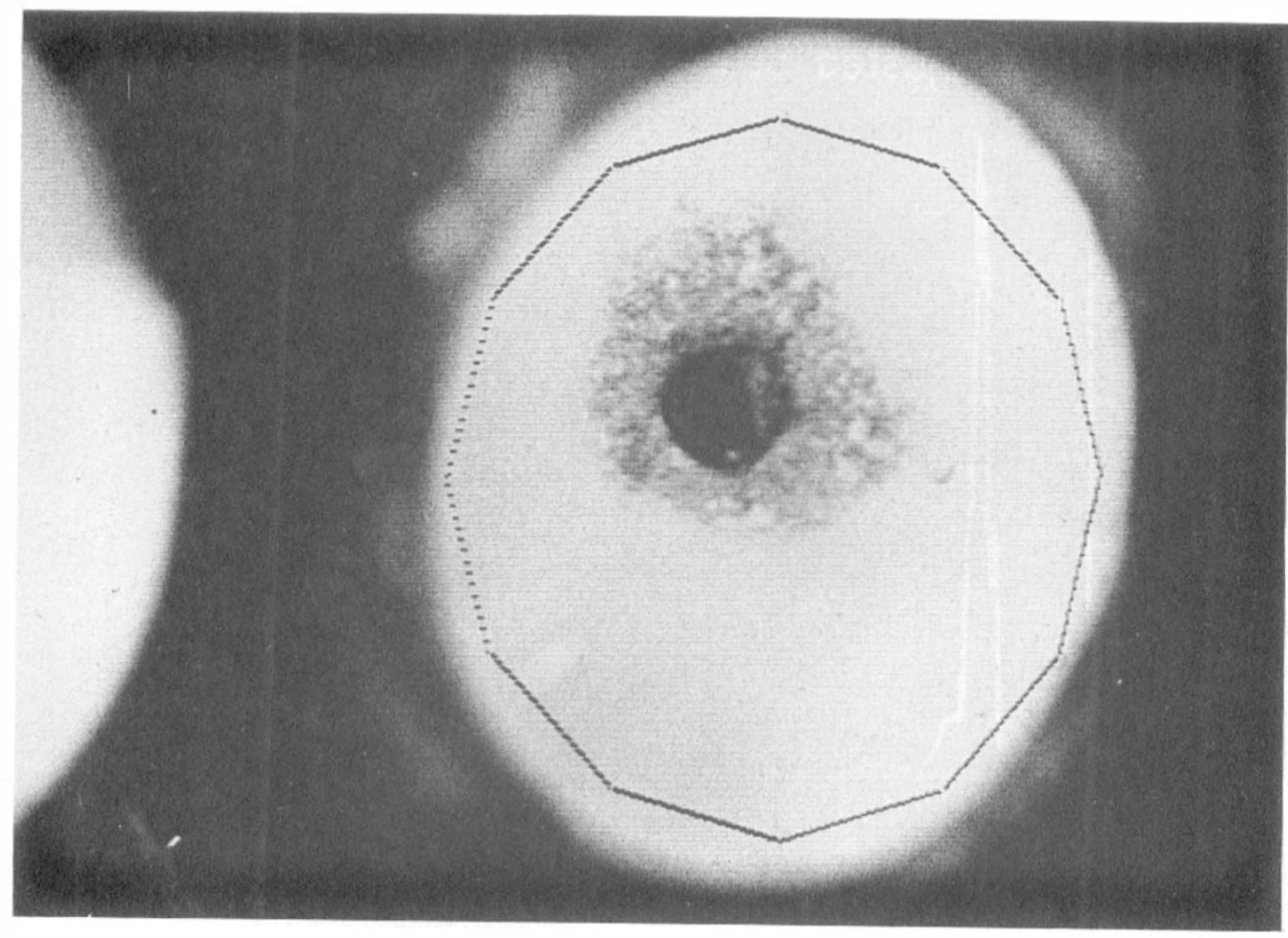

Fig. 5. Retro-illumination image of posterior sub-capsular cataract as seen on the video screen, with circular area of interest indicated as a polygon superimposed on the image. (The actual analysis uses a 'true' circle, the polygon being an approximation which permits rapid display when choosing the circular 'area of interest'). The circle size is calibrated to refer to the original dimensions of the object.

posterior projection is occupied by cortical and sub-capsular cataract?

(ii) What is their apparent optical density in retro-illumination?

In the analysis of retro-illumination images there are four main problems to overcome: Firstly, how to deal with variable sized pupils with variable amounts of lens (and cataract) being visible. Secondly how to account for variability in illumination, ie. photographic variability and flash intensity variability in photographic systems, variability of the light source for the CCD cameras, variability of the amount of light entering the eye through different sized pupils, and variability of the amount of light reflected off the retina in the retro-illumination image. Thirdly, how to decide the cut-off level between cataract and clear lens. Fourthly in longitudinal studies where sequential images of the same cataract are to be compared there are problems relating to alignment of serial images.
To standardise the area of pupil to be analysed, a discrete series of circles are available to the operator (Fig. 5). (To speed presentation these appear on the screen as polygons although the actual analysis is performed on 'true' circles.) The sizes of these circles refer to apparent size of the pupil (ignoring corneal magnification) via a calibration system, and varies in steps of $0.5 \mathrm{~mm}$. For a particular study, a standard analysis diameter of say, $8 \mathrm{~mm}$ or $7.5 \mathrm{~mm}$ may be selected for all images in the series. The circular 'area of interest' thus defined is used for the subsequent digital analysis.

In the past, the variability in lighting levels and photographic development have severely limited the repeatability of measures derived from image analysis of retro-illumination photographs of the lens. ${ }^{16} \mathrm{~A}$ major source of variability results from minor changes in the alignment of the eye, which result in the optic nerve moving in and out of position as the 


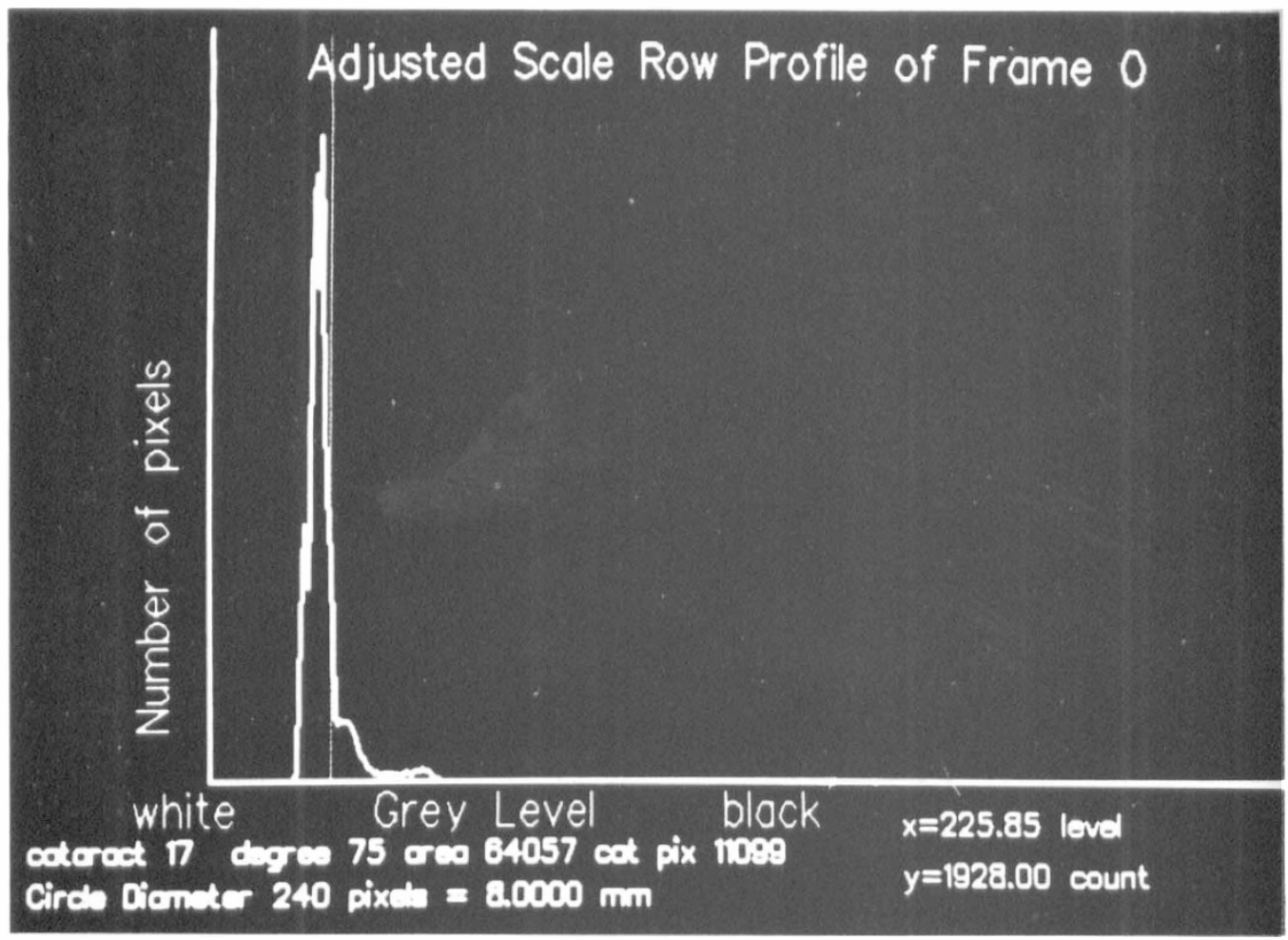

Fig. 6. Screen display of the histogram of pixels across the grey scale for an area analysis of the circular 'area of interest' as defined for the Retro-illumination image seen in Fig. 5. Darker pixels (corresponding to cataractous regions of the lens) occupy the 'right skewed' tail of the histogram. The cut-off point between cataract and clearlens is determined automatically by the computer program, and the position of the cursor indicates graphically the position of this cut-off. The percentage cataract (17\%) and the cataract 'degree' (75 units) are indicated below.

main 'reflector' of light for the retro-illumination image. Our camera systems diminish this variability on a 'within patient' basis by providing fixed fixation lights for the subject during image acquisition. ${ }^{13}$ This problem has been further overcome by performing a digital transform on the frequency distribution of the grey levels within the 'area of interest'. This standardises the frequency distribution, and dramatically decreases the loss of repeatability due to variability in illumination levels and photographic development.

A density threshold between cataract and clear lens was defined by making a series of measurements on clear lenses to establish the range of spread of standardised histograms from non-cataractous lenses from individuals of various ages. The threshold was set, such that truly clear lenses would be correctly identified as non-cataractous $98 \%$ of the time. This approach means that certain minor opac- ities may be missed by the analysis, but the

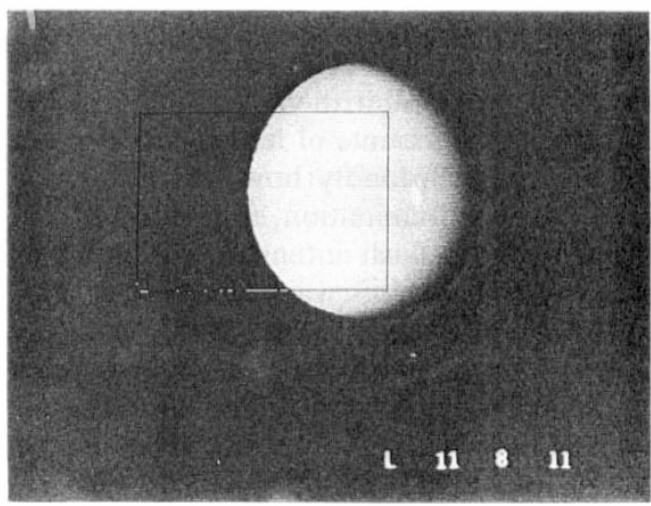

Fig. 7. Auto-fluorescence image of the crystallinelens as seen on the video screen with rectangular 'area of interest'. The area is chosen to include a region of fluorescing lens and a background region (analysis shown in Fig. 8). 
specificity for the identification of opacification is high.

Serial alignment of images is in general ensured by the fixation lights on our retroillumination camera. ${ }^{13}$ Selection of the central area within the pupillary aperture as the area of interest further ensures that the regions of cataract and clear lens being examined digitally are consistently the same within an individual eye in a longitudinal assessment. The potential for errors from this source is greatest when the opacities extend to the periphery of the pupil.

The percentage of the specified area of interest occupied by cataract is thus available, and is automatically determined by the computer (Fig. 6). To take account of the apparent density of the opacities present, a 'weighted integral' of the histogram is used, which weights preferentially optically dense opacities according to their grey levels. This quantity, referred to as the 'degree of cataract density' is calculated as the sum of the products of the number of pixels at a particular grey level (height of the histogram) by the distance above threshold for that grey level. This quantity provides a summary statement covering both the area of opacity as well as the apparent density of opacity.

Two indices of cortical/sub-capsular opacification are thus available, one is a simple percentage measure (ratio), while the other is a combined area and density measure (units arbitrary, Fig. 6). User monitoring of analyses is employed to detect unusual situations where the computer might otherwise generate errors. This situation may for example arise when there is a low spatial frequency density gradient across an image resulting from malalignment of the eye during image acqui-

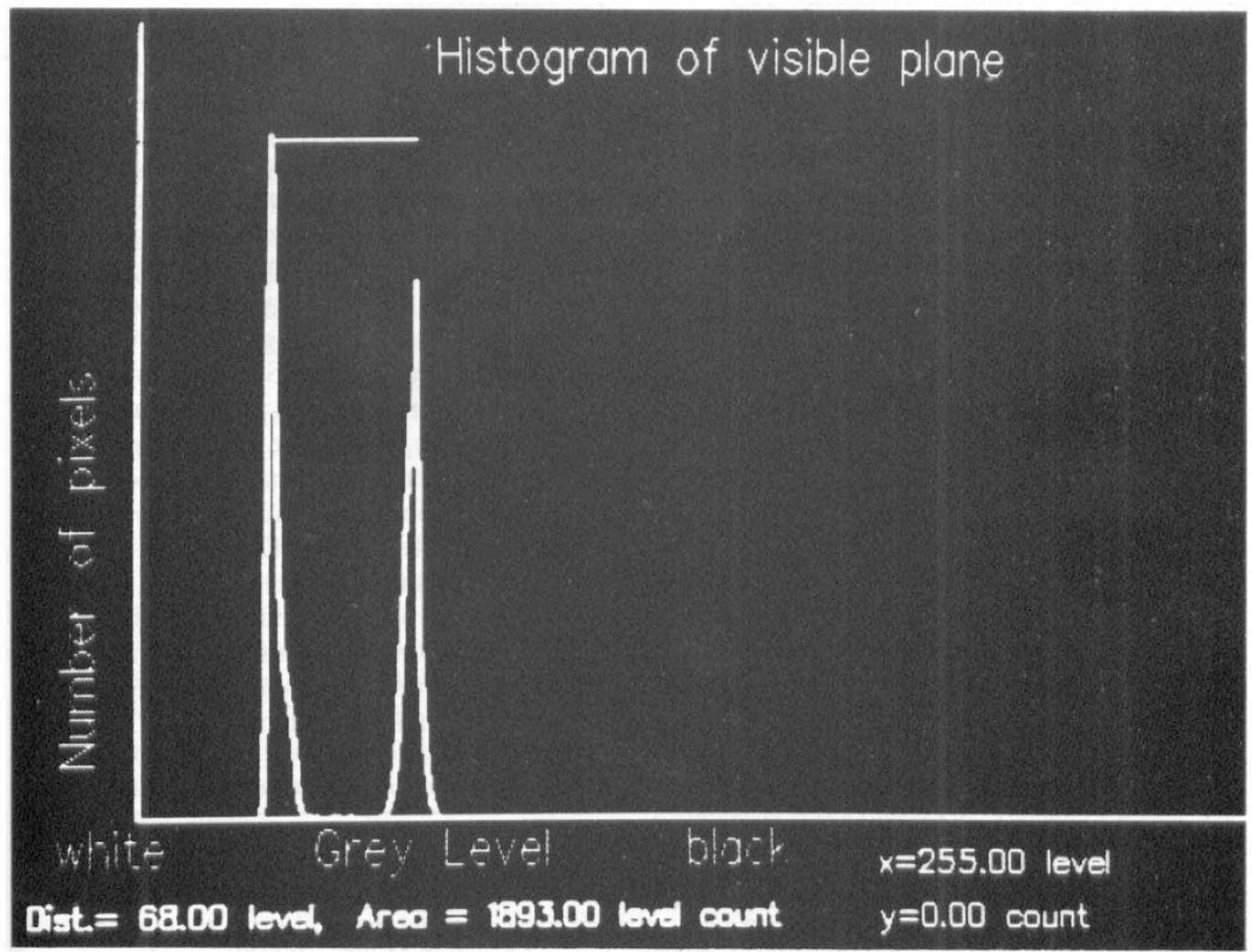

Fig. 8. Screen display of the histogram of pixels across the grey scale for an area analysis of the rectangular' area of interest' as defined for the lenticular auto-fluorescence image seen in Fig. 7. The two peaks represent the region of lenticular autofluorescence and the background respectively. The 'distance' between the foreground fluorescence peak and background peak on the standardised histogram is used as the measure of auto-fluorescence (68 Fl units, scale arbitrary. 
Table I Analysis of errors for Scheimpflug, and auto-fluorescence measures.

\begin{tabular}{llc}
\hline Feature & 0.95 Prob. Error Range & $\%$ of Range \\
\hline Nuclear scatter & $\pm 0.43 \mathrm{ND}$ & $\pm 4 \%$ \\
Lens thickness & $\pm 0.067 \mathrm{~mm}$ & $\pm 2 \%$ \\
Anterior clear zone & $\pm 0.028 \mathrm{~mm}$ & $\pm 8 \%$ \\
Nuclear size & $\pm 0.089 \mathrm{~mm}$ & $\pm 8 \%$ \\
Cortical thickness & $\pm 0.112 \mathrm{~mm}$ & $\pm 3 \%$ \\
Front curve & $\pm 0.61 \mathrm{~mm}$ & $\pm 5 \%$ \\
Back curve & $\pm 1.62 \mathrm{~mm}$ & $\pm 30 \%$ \\
Anterior chamber depth & $\pm 0.038 \mathrm{~mm}$ & $\pm 1 \%$ \\
Auto-fluorescence & $\pm 0.15 \mathrm{Fl} \mathrm{units}$ & $\pm 13 \%$ \\
\hline
\end{tabular}

KEY: $\quad$ Feature $=$ Feature of lens being measured; 0.95 Prob. Error Range $=$ range within which there is a probability of 0.95 that any individual error will fall in the population under study; \% of Range = Error range as percentage of dynamic range $(\max -\min )$ of the measure under consideration; ND = neutral density units; FL units $=$ Units of auto-fluorescence (arbitrary scale).

sition. Such 'poor quality' images may be unsuitable for analysis and user monitoring is required to discard poor images.

\section{(3) Digital Analysis of Auto-fluorescence Images}

The intensity of lenticular auto-fluorescence is measured by examining digitally a rectangular sample of grey levels from the autofluorescence image as shown in Figure 7, which is an example of blue/green fluorescence. The rectangular area of interest which is analysed includes part of the fluorescing lens and part of the background of the image, care being taken to avoid the specular corneal reflex. The frequency distribution of the pixels across the grey scale within the rectangular 'area of interest' produces a bimodal distribution (Fig. 8). The 'white peak' represents the fluorescing lens, while the 'dark peak' represents the background. As with the retroillumination system, the histogram is standardised. The measure used for the intensity of the auto-fluorescence is the difference between the two peaks of the standardised histogram. The units of fluorescence are arbitrary, with a total range of between 0 and 130 units.

\section{Repeatability of the Methods}

Repeatability was assessed in a pilot study for Scheimpflug, retro-ilumination, and fluorescence images. The pilot study examined the repeatability of images obtained using the photographic camera systems. Repeat images were examined: forty Scheimpflug images from ten eyes (four each), sixty retro-illumination images from thirty eyes (two each), and sixty fluorescence images from thirty eyes (two each). The series of Scheimpflug images for each eye was obtained at the same photographic session, while the retro-illumination and fluorescence repeat images were obtained at different photographic sessions several months apart (range 6 to 12 months). In each case the photographer was the same (JS).

Populations of differences away from the mean value were examined statistically. Where these populations of differences were found to be Gaussian, errors were expressed

Table II Non-parametric analysis of the repeatability of the retro-illumination cataract indices.

\begin{tabular}{lll}
\hline Test & $\%$ Cataract & Degree of Cataract Density \\
\hline Spearman Corr & $0.97(P<0.0001)$ & $0.89(P<0.0001)$ \\
Mann-Whitney & $P=0.79$ & $P=0.57$ \\
Kappa value & $0.94(\mathrm{SE}=0.008)$ & $0.54(\mathrm{SE}=0.017)$ \\
\% Exact & $93 \%$ & $73 \%$ \\
\hline
\end{tabular}

KEY: Spearman Corr = Spearman rank correlation coefficient; Mann-Whitney = Mann-Whitney U test for differences between groups (none found); Kappa value = Kappa statistic (chance corrected measure of agreement); \% Exact $=$ Percentage exact agreement in groups as re-partitioned for the Kappa analysis (see text). 
Table III Analysis of errors for retro-illumination measures.

\begin{tabular}{llll}
\hline Feature & $\%$ of Errors & Error Range & \% of Range \\
\hline Percentage cataract & $93 \%$ & $\pm 1 \%$ & $\pm 1 \%$ \\
Degree cataract density & $87 \%$ & \pm 2 units & $\pm 0.5 \%$ \\
\hline
\end{tabular}

KEY: $\quad$ Feature $=$ Feature of lens being measured; $\%$ of Errors $=$ the percentage of the errors which fell into the stated error range; Error Range = range within which the stated percentage of individual errors fell; \% of Range $=$ Error range as percentage of dynamic range $(\max -\min )$ of the measure under consideration .

as the range within which there is a probability of 0.95 that any individual error away from the mean will fall. This range was also expressed as a percentage of the dynamic range (maximum-minimum value) of the particular measure under consideration. These ranges are given in Table I. Where the error distribution was non-Gaussian (as in retroillumination errors) a number of non-parametric measures of agreement were used. Spearman rank correlation coefficient and the Mann-Whitney U-test for correlation and differences respectively between initial and subsequent measures were performed. Weighted Kappa and percentage exact agreement were determined by dividing the nonzero range of errors into quartiles, with zero error constituting a fifth class of error. A final analysis examined ranges of errors broadly comparable to those described above in the parametric analysis. These were tabulated for arbitrarily chosen percentage ranges. The non-parametric analyses for retro-illumination errors are tabulated in Tables II and III.

\section{Discussion}

The extraction of statistically analysable data from images of the crystalline lens is central to the study of lens features and lens opacification. In order to facilitate such study, a computerised digital image analysis system has been developed. The methods developed include lens biometry, quantification of cataract, and measurement of lenticular auto-fluorescence.

The system described is capable of analysis of Scheimpflug, retro-illumination and fluorescence images, and is modular, allowing the user to choose between photographic and direct CCD image acquisition. The system is sufficiently versatile to allow photographs from various cameras to be analysed.

The pilot study of the repeatability of the system with reference to analysis of photographically derived images indicates an acceptable level of repeatability for most of the measures assessed. The experimental design for the repeatability study was not uniform, repeat Scheimpflug images were obtained at the same photographic session, while repeat retro-illumination and fluorescence images were obtained on separate occasions several months apart. The Scheimpflug data may therefore slightly under-estimate the variability, whereas for the other two image types the variability may be over-estimated. (Individual lenses may have changed between the initial and subsequent assessments). ${ }^{28}$ Although the pilot study results using photographically based methods are encouraging, further repeatability assessments are necessary fully to define the variability of this new technology. Further studies are presently underway to address this issue with regard to the entire system, including the new generation of direct input CCD cameras.

\section{References}

${ }^{1}$ Kahn HA, Leibowitz H, Ganley PJ, Kini M, Colton T, Nickerson R, Dawber TR: Standardizing diagnostic procedures. Am J Ophthalmol 1975; 79: 768-75.

${ }^{2}$ Sommer A: Cataracts as an epidemiologic problem. Am J Ophthalmol 1977, 83: 334-9.

${ }^{3}$ Sasaki K, Oishi T, Yamaaki H, Nakamura F: Documentation of human lens by rotating photoslitlamp. Clinical applications and examinations for the reproducibility of obtained photographs. Jpn J Ophthalmol 1979, 33: 621-7.

${ }^{4}$ Leibowitz HM Krueger DE, Maunder LR et al: The Framingham eye study monograph. Survey Ophthalmol 1980, 24: (Suppl), 336-610.

${ }^{5}$ Duncan G: On classifying human cataractous lenses. In: Mechanisms of cataract formation in the human lens. (1981). Ed: Duncan G. Academic Press, London. P:1-5.

${ }^{6}$ Leske MC, Sperduto RD: Epidemiology of senile cataracts: A review. Am J Epidemiol 1983, 118: 152-65.

${ }^{7}$ Chylack LT: Classification of human cataractous 
change by the American co-operative cataract research group method. In: Human cataract formation. [Ed: Nugent J, Whelan J] Pitman, London. (CIBA Foundation Symposium, 106). P: 3-24. (1984).

${ }^{8}$ Sparrow JM, Ayliffe W, Bron AJ, Brown NAP, Hill AR: Inter-observer and intra-observer variability of the Oxford Clinical Cataract Classification and Grading System. Internat Ophthalmol 1988, 11: 151-7.

${ }^{9}$ Brown NAP: Quantitative slit-image photography of the lens. Trans Ophthalmol Soc UK 1972, 92: 303-17.

${ }^{10}$ Hockwin O, Dragomirescu V, Laser H: Measurements of lens transparency or its disturbances by densitometric image analysis of Scheimpflug photographs. Graefe's A rch Clin Exp Ophthalmol 1982, 219: 255-62.

${ }^{11}$ Kawara T and Obazawa $\mathrm{H}$ : A new method for retroillumination photography of cataractous lens opacities. Am J Ophthalmol 1980, 90: 186-9.

${ }^{12}$ Brown NAP, Bron AJ, Ayliffe W, Sparrow JM, Hill AR: the Objective Assessment of Cataract. Eye 1987, 1: 234-46.

${ }^{1.3}$ Brown NAP: The Oxford Retro-Illumination Cataract Recording Camera-a new instrument. $J$ Audiovisual Media in Medicine 1988, 11: 58-60.

${ }^{14}$ Kawara T, Obazawa H, Nakano R, Sasaki M, Sakata T: Quantatative evaluation of cataractous lens opacities with retro-illumination photography. Jpn J Ophthalmol 1979, 33: 21-6.

${ }^{15}$ Maclean H, Taylor CJ: An objective staging for cortical cataract in vivo aided by pattern-analysing computer. Exp Eye Res 1981, 33: 597-602.

${ }^{16}$ Chylack LT Jr, Rosner B, Cheng HM et al: Sources of variance in the objective documentation of human cataractous change with Topcon SL-45 and Neitz-CTR retroillumination photography and computerized image analysis. Curr Eye Res 1987, 6: 1381-9.

${ }^{17}$ Hannah $\mathrm{K}$ and Tarassenko L: Tracking cataract by the four-line method. Image and Vision Computing 1989, 7: 57-62.

${ }^{18}$ Wolfe JR and Chylack LT: Objective analysis of percent opacification in retro-illumination lens photographs. Invest Ophthalmol [suppl] (ARVO) 1989, 30: 328.

${ }^{14}$ Shun-Shin GA, Hannah K, Bron AJ, Brown NAP: A new index of cataract progression. Invest Ophthalmol (ARVO) (Suppl) 30: 329.

${ }^{20}$ Helve $\mathrm{J}$ and Nieminen H: Autoflouorescence of the human diabetic lens in vivo. Am J Ophthalmol 1976, 81: 491-4.

${ }^{21}$ Lerman S and Hockwin O: Ultraviolet-visible slit lamp densitography of the human eye. Exp Eye Res 1981, 33: 587-96.

"2hibata T and Sasaki K: Observation of Ageing Changes of Lens Transparency-Analysis of 541 eyes from Color Images. (1982) 86: 1701-8.

${ }^{23}$ Sasaki K, Hiiragi M, Sakamoto Y, Shibato T: In vivo colour analysis of human crystalline lenses. Ophthalmic Res 1985, 17: 21-6.

${ }^{24}$ Brown NAP: Slit-image photography. Trans Ophthalmol Soc UK 1969, 89: 397-408.

${ }^{25}$ Brown NAP: An advanced slit image camera. $\mathrm{Br} J$ Ophthalmol 1972, 56: 624-31.

${ }^{26}$ Sparrow JM: The Lens in Diabetes. D Phil Thesis, Linacre College, University of Oxford. (1988)

${ }^{27}$ Brown NAP: Biometry of the lens of the eye. MD Thesis, University of Cambridge. (1975).

${ }^{28}$ Shun-Shin GA, Brown NAP, Bron AJ, Sparrow JM: The dynamic nature of posterior sub-capsular cataract. Br J Ophthalmol 1989, 73: 522-7. 